\title{
An Unexpected Pregnancy Test Result Leading to the Diagnosis of Advanced Gastric Cancer
}

\author{
Jordonna Brown'1, Odeth Barrett-Campbell ${ }^{1}$, Yakira David ${ }^{1}$, Carol Luhrs², Samy I. McFarlane, ${ }^{1, *}$ \\ ${ }^{1}$ Division of Medicine, SUNY Downstate Medical Center, Brooklyn, NY 11203, USA \\ ${ }^{2}$ Division of Hematology and Oncology, Veterans Affairs New York Harbor Healthcare System, Brooklyn, NY 11209, USA \\ *Corresponding author: Samy.mcfarlane@downstate.edu
}

Received May 10, 2019; Revised June 18, 2019; Accepted July 05, 2019

\begin{abstract}
The presence of beta human chorionic gonadotropin in the absence of pregnancy warrants further workup as elevated levels may be seen in trophoblastic disease or germ cell tumors and rarely in solid tumors such as gastric cancers. The signs and symptoms of gastric cancer are vague and as such, the diagnosis is often delayed. Overexpression of the beta-subunit of human chorionic gonadotrophin may be seen in close to $50 \%$ of gastric cancers. The implication of this finding is unclear; however, beta human chorionic gonadotropin reactivity may play a role in tumorigenesis and often correlates with an advanced stage at presentation. Herein, we present a case report of a patient with an incidental finding of a positive urinary human chorionic gonadotropin test which led to the diagnosis of advanced gastric cancer.
\end{abstract}

Keywords: gastric cancer, tumor markers, $\beta H C G$, non-pregnant

Cite This Article: Jordonna Brown, Odeth Barrett-Campbell, Yakira David, Carol Luhrs, and Samy I. McFarlane, "An Unexpected Pregnancy Test Result Leading to the Diagnosis of Advanced Gastric Cancer." American Journal of Medical Case Reports, vol. 7, no. 7 (2019): 148-150. doi: 10.12691/ajmcr-7-7-8.

\section{Introduction}

Gastric cancer is one of the leading causes of cancer-related deaths in the world and a significant contributor of morbidity. The initial symptoms are often vague which delays diagnosis. In fact, the symptoms of unexplained weight loss, epigastric bloating, early satiety and persistent vomiting usually represent locally advanced or widespread metastatic disease [1].

The use of tumor markers such as carbohydrate antigen 19-9 (CA 19-9), carcinoembryonic antigen (CEA), cancer antigen 125 (CA-125) and cancer antigen 72-4 (CA 72-4) have been suggested for pre and post-operative monitoring of the evaluation of resection and/or recurrence [2]. The combination of these markers has been shown to increase diagnostic sensitivity. There have also been emerging studies which show that close to $50 \%$ of gastric cancers may express the beta-subunit of human chorionic gonadotropin $(\beta \mathrm{HCG})$, a tumor marker that may correlate with a high tumor burden. In the absence of pregnancy and trophoblastic tumors, elevated $\beta \mathrm{HCG}$ is ominous for malignancies such as osteosarcoma, testicular seminoma pancreatic cancer and gastric carcinoma [3]. Here we present the case of a non-pregnant woman with gastric carcinoma who was diagnosed after incidental finding of a positive $\beta \mathrm{HCG}$.

\section{Case Presentation}

A 47-year-old Afro-Caribbean female with a past medical history of uterine fibroids presented with vague symptoms of intermittent abdominal pain, nausea and vomiting for two weeks. Her last menstrual period was one week prior to presentation and the patient reported being celibate for the past 4 years. She denied weight loss, early satiety or change in bowel habit. The rest of the history was unremarkable. The initial examination revealed normal vital signs and generalized abdominal tenderness without rebound or guarding. Lab investigations were significant for hemoglobin $8(12-15 \mathrm{~g} / \mathrm{dL})$, MCV 80.4 (80-96 fL/red cell), Iron 14 (40-155ug/dl), TIBC 265 (240- $450 \mathrm{ug} / \mathrm{dl})$ and Ferritin 9.9 (12-150ng/dl), alkaline phosphatase 476 (40-100 U/L) and a positive urine $\beta \mathrm{HCG}$. The renal indices and other hepatic function tests were within normal limits. A transabdominal ultrasound showed an intramural uterine density and no evidence of an intrauterine pregnancy. Serum $\beta \mathrm{hCG}$ was $75.0(0-5 \mathrm{mIU} / \mathrm{ml})$.

The patient was referred to the gynecology service who determined that pregnancy was unlikely and ordered a CT abdomen-pelvis to evaluate the density in the uterus. This study revealed multiple scattered retroperitoneal, celiac axis, gastrohepatic ligament and pelvic lymph nodes measuring up to $1.4 \mathrm{~cm}$ in short axis suspicious for metastatic disease or lymphoma. It also showed ascites, 2 small 
uterine fibroids, ill-defined heterogenous density along uterine fundus and a $1.9 \mathrm{~cm}$ soft tissue nodule adjacent to the spleen. The imaged osseous structures appeared sclerotic.

Histological examination of a biopsied left para-aortic lymph node showed poorly differentiated metastatic adenocarcinoma with signet cell features suggestive of a gastric primary [Figure 1]. Subsequent esophagogastroduodenoscopy was remarkable for irregular gastric mucosa, biopsy of which revealed poorly differentiated adenocarcinoma [Figure 2]. HER-2 receptors were negative and there was no evidence of Helicobacter Pylori infection.

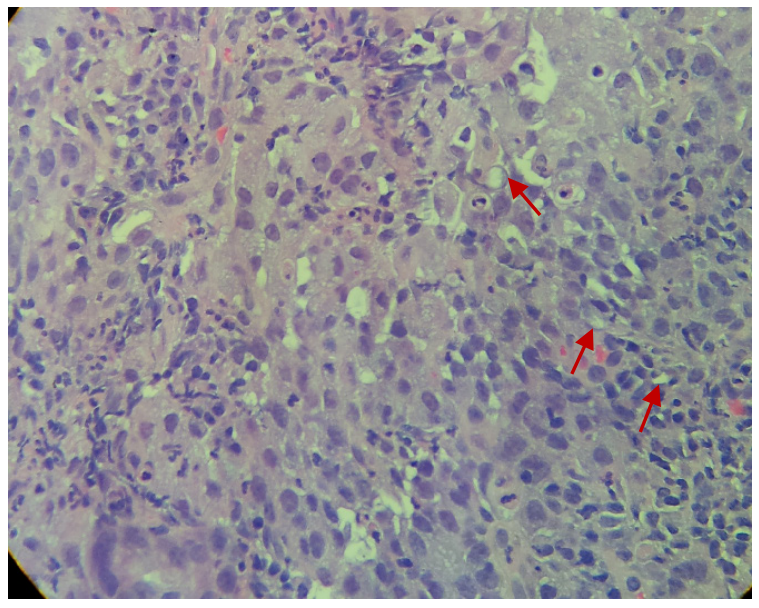

Figure 1. H\&E stain of left para-aortic lymph node. High power magnification showing poorly differentiated metastatic adenocarcinoma with $<50 \%$ signet ring features (red arrows)

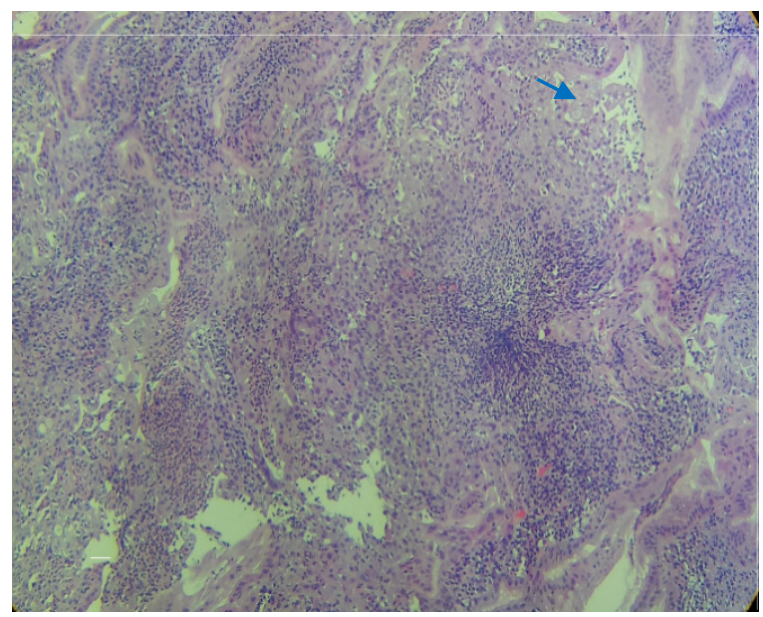

Figure 2. H\&E stain of gastric mucosa. Low power magnification showing solid sheets of tumor cells (poorly differentiated) with minimal gland formation (blue arrow)

The patient was initiated on Cisplatin and 5-Fluorouracil and after 2 cycles had transient improvement of her $\beta \mathrm{HCG}$ levels to $45(0-5 \mathrm{mIU} / \mathrm{ml})$. She subsequently developed recurrent exudative pleural effusions with cytology that was negative for malignancy. Although cytology was negative, the effusions were thought to be due to gastric cancer. Profound thrombocytopenia and pulmonary hemorrhage then developed, and she had cardiac arrest. The patient passed away within 3 months of initial diagnosis.

\section{Discussion}

Gastric cancer is the fifth most common cancer worldwide with an estimated incidence of 952,000 cases in 2012 [4]. Approximately $70 \%$ of these cases occur in developing countries with a strong preponderance in East Asia. This cancer still carries a high mortality and is the $3^{\text {rd }}$ most lethal cancer with mortality rates of 723,000 [4]. Patients with gastric cancer are often asymptomatic in the early stages when resection of the primary lesion could effect cure. Instead, most patients present with advanced or widely metastatic disease which may manifest with symptoms of unintentional weight loss, early satiety, abdominal bloating or distention and gastrointestinal bleed from an ulcerated lesion [1]. Several risk factors, including Helicobacter pylori infection, family history of gastric cancer, history of chronic atrophic gastritis, smoking, heavy alcohol consumption, obesity and low socioeconomic status, may contribute to the development of gastric cancer $[1,5]$. Of these, Helicobacter pylori infection has been found to be most closely related to the occurrence of gastric cancer. The process appears to be multifactorial and involves host and environmental factors. There has however been a decline in the prevalence of Helicobacter Pylori in some developed countries due to improved hygiene and food preparation as well as increased use of antibiotics [5].

There are currently no specific tumor markers that have adequate sensitivity or specificity for diagnosis or follow-up of gastric cancer. However, serum levels of CEA, CA 125, CA 19-9, CA 72-4 and even less commonly $\beta$ hCG may be elevated in patients with gastric cancer and are associated with a poorer prognosis [2]. However, the use of these of markers have not been standardized in clinical practice. Human chorionic gonadotropin is normally produced by the syncytiotrophoblast cells of the placenta and is used to detect and monitor pregnancy. The beta-subunit of hCG is a sensitive and specific marker for trophoblastic tumors of placental and germ cell origin [6]. It can also be falsely elevated in hypogonadal states, exogenous administration and marijuana use. Outside of these contexts elevated levels of $\beta \mathrm{hCG}$ are usually associated with malignancies such as breast cancer, leiomyosarcoma, pancreatic cancer, lymphoma and leukemia [6]. It may also be elevated in other malignancies, such as gastric cancer, with an undefined sensitivity or specificity [2]. The role of $\beta \mathrm{hCG}$ in the progression of gastric cancer remains unclear but its presence is usually consistent with a more poorly differentiated tumor and associated worse prognosis $[3,6]$.

In evaluating patients with elevated $\beta$ hCG levels, work-up for malignancy must be performed once pregnancy is ruled out. The work-up should not be limited to the tumors that are conventionally associated with elevated $\beta$ hCG but should also be expanded to include a broad base of organs. This approach is strongly warranted as the presence of this hormone may signify the presence of an insidious but aggressive malignancy and has significant prognostic implications. 


\section{Acknowledgements}

The authors would like to thank the pathology department of Kings County Hospital Center for contributing the histology slides.

This work is supported, in part, by the efforts of Dr. Moro O. Salifu M.D., M.P.H., M.B.A., M.A.C.P., Professor and Chairman of Medicine through NIH Grant number S21MD012474.

\section{Conflicts of Interests}

The authors declare that there are no conflicts of interests.

\section{Funding}

This research received no specific grant from any funding agency in the public, commercial, or not-for-profit sectors.

\section{References}

[1] Zali H, Rezaei-Tavirani M \& Azodi M: Gastric cancer: Prevention, risk factors and treatment. Gastroenterology and Hepatology from Bed to Bench 2011; 4 (4): 175-185.

[2] Jiexian J, Xiaoqin X, Lili D, Baoguo T, Ting S, Xianwen Z \& Cunzhi H: Clinical assessment and prognostic evaluation of tumor markers in patients with gastric cancer. International Journal of Biological Markers 2013; 28 (2): 192-200.

[3] Birkenfeld, S, Noiman, G, Krispin, M, Schwartz, S, Zakut, H: The incidence and significance of serum hCG and CEA in patients with gastrointestinal malignant tumors. European Journal of Surgical Oncology 1989; 15 (2): 103-8.

[4] World Health Organization. Stomach cancer estimated incidence, mortality and prevalence worldwide in 2012,

http://globocan.iarc.fr/Pages/fact_sheets_cancer.aspx (n.d)

[5] Blaser MJ. Hypothesis: The changing relationships of Helicobacter pylori and humans: implications for health and disease. J Infect Dis 1999; 179(6): 1523-30.

[6] Walker L \& Erler B: Gastric cancer in the setting of Persistently elevated human chorionic gonadotropin: A case report. Case Reports in Obstetrics and Gynecology, 2011; 2011: 1-4.

C The Author(s) 2019. This article is an open access article distributed under the terms and conditions of the Creative Commons Attribution (CC BY) license (http://creativecommons.org/licenses/by/4.0/). 